\title{
Modelación de la presencia de Euphorbia antisyphilitica Zucc mediante propiedades físicas y químicas del suelo
}

\section{Modelation of the presence of Euphorbia antisyphilitica Zucc with physical and chemical properties of soil}

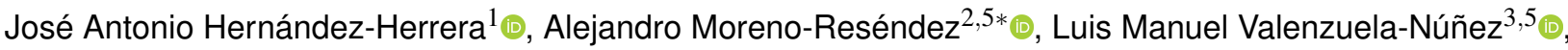 \\ Martín Martínez-Salvador ${ }^{4}$ \\ ${ }^{1}$ Estudiante de Doctorado del Programa de Posgrado en Ciencias en Producción Agropecuaria. Universidad Autónoma Agraria Antonio \\ Narro, Periférico Raúl López Sánchez s/n. Colonia Valle Verde, CP. 27054. Torreón, Coahuila, México. \\ ${ }^{2}$ Universidad Autónoma Agraria Antonio Narro, Periférico Raúl López Sánchez s/n. Colonia Valle Verde, CP. 27054. Torreón, Coahuila, \\ México. \\ ${ }^{3}$ Universidad Juárez del Estado de Durango. Facultad de Ciencias Biológicas. Avenida Universidad s/n. Fracc. Filadelfia, CP. 35010. \\ Gómez Palacio, Durango, México. \\ ${ }^{4}$ Universidad Autónoma de Chihuahua. Facultad de Zootecnia y Ecología. Periférico Francisco R. Almada Km 1, CP. 31453. Chihuahua, \\ Chihuahua, México. \\ ${ }^{5}$ Integrante de la Red Académica de Innovación en Alimentos y Agricultura Sustentable (RAIAAS) - Consejo Estatal de Ciencia y \\ Tecnología (COECYT) y La Comunidad de Instituciones de Educación Superior de la Laguna (CIESLAG). \\ *Autor de correspondencia: alejamorsa@yahoo.com.mx y alejamorsa@hotmail.com
}

Artículo científico recibido: 10 de julio de 2018 aceptado: 29 de mayo de 2019

RESUMEN. México es el único productor y exportador a nivel mundial de la cera de candelilla, pero el mercado internacional se establecieron restricciones con la finalidad de disminuir la presión sobre el recurso natural por la sobreexplotación, por lo que actualmente se encuentra incluida en la lista de la Convención Internacional sobre el Comercio de Especies Amenazadas de Fauna y Flora (CITES). El objetivo fue identificar las propiedades del suelo que favorecen la distribución de la candelilla, mediante el método de máxima entropía, buscando promover su manejo y aprovechamiento sustentable. El área de estudio fueron dos localidades de los municipios de Cuatro Ciénegas y Viesca, Coahuila. Se obtuvieron 102 y 72 sitios de muestreo en los que se registraron las coordenadas, los datos biométricos de los ejemplares, las condiciones del hábitat, del relieve y del tipo de suelo. Se seleccionaron nueve propiedades edáficas, para lo que se descargaron los archivos de la base de datos Soilgrid, la modelación se realizó en el programa Maxent. Como resultados el área bajo la curva en cada localidad con valores 0.927 y 0.973 , se considera como un modelo adecuado para determinar la distribución actual de la especie. En el suelo, los factores físicos que fueron determinantes para favorecer la presencia de candelilla fueron: densidad aparente, profundidad y pedregosidad, mientras que los factores químicos: capacidad de intercambio catiónico y el contenido de carbono orgánico.

Palabras clave: Candelilla, cerote, distribución, entropía, modelos de predicción, zonas áridas.

ABSTRACT. Mexico is the only producer and exporter of candelilla wax in the world. International restrictions were established in the international market in order to reduce the pressure on the natural resource due to overexploitation; currently, this specie is included in the list of Convention on International Trade in Endangered Species of Wild Fauna and Flora (CITES). The objective was to identify soil properties that favor candelilla distribution, using maximum entropy method, seeking to promote its management and sustainable use. Study area was established in two locations in the municipalities of Cuatro Ciénegas and Viesca, Coahuila. We obtained 102 and 72 sampling sites in which coordinates were registered, specimens biometric data, habitat conditions, relief and soil type. Nine edaphic properties were selected, which the files of the Soilgrid database were downloaded and the modeling was made in the Maxent program. As a result, area under the curve in each locality with values 0.927 and 0.973 is considered as an adequate model to determine current distribution of specie. In the soil, the physical factors that were decisive to favor the presence of candelilla were: depth, bulk density and stoniness, while 
the chemical factors were: cation exchange capacity and organic carbon content.

Key words: Candelilla, wax, distribution, entropy, prediction models, arid zones.

\section{INTRODUCCIÓN}

El aprovechamiento de las poblaciones de Euphorbia antisyphilitica Zucc, conocida como candelilla, tiene diferentes implicaciones, principalmente de tipo ecológico, económico y social, ya que su actividad productiva genera empleos e ingresos monetarios con la comercialización de la materia prima (Arato et al. 2014). Respecto a su categoría ecológica, es una especie con respuesta positiva a las condiciones adversas del ambiente, tiene tolerancia al estrés hídrico, y las altas y bajas temperaturas (Martínez-Ballesté y Mandujano 2013, Muñoz-Ruiz et al. 2016). Se desarrolla en condiciones restrictivas del suelo como $\mathrm{pH}$ de ligeramente ácido a alcalino (6.3 a 8.4), presencia de material geológico como rocas, preferentemente de carbonato de calcio, en suelos con profundidad menor a 30 $\mathrm{cm}$, textura franco-arenosa, con buen drenaje, estructura granular, presencia de piedras y bajo contenido de materia orgánica de 0.96 a 2.64\% (ZamoraMartínez et al. 2013). Por lo que es una de las especies con mayor adaptación a las condiciones de las zonas áridas, ya que tiene mecanismos que le permiten sobrevivir y reproducirse en condiciones de escasa disponibilidad de agua y temperaturas extremas (Rojas-Molina et al. 2011). Es nativa de las zonas áridas y semiáridas del norte de México donde la temperatura media anual es superior a los $20^{\circ} \mathrm{C}$ y la precipitación oscila entre 50 y $350 \mathrm{~mm}$ anuales. Debido a estos requerimientos posee una amplia distribución en los estados de Durango, Chihuahua, Coahuila, Nuevo León, San Luis Potosí, Tamaulipas y Zacatecas. Siendo Coahuila el estado más importante, aportando aproximadamente el $80 \%$ de la producción nacional (Villa-Castorena et al. 2010).

En el manejo forestal es indispensable conocer la distribución de las especies y se considera que la modelación es una herramienta adecuada para llevar a cabo esta actividad, hay varias metodologías, destacando la de nicho ecológico, la cual usa un procedimiento correlativo y sencillo, que requiere de información sobre la presencia de cada especie, integrado a los parámetros del clima y suelo que favorecen la presencia de la especie de interés (Phillips et al. 2006, Owens et al. 2013). Lo que permite entender la combinación de los factores ambientales que favorecen la especie estudiada en una región geográfica y los efectos en los factores ambientales (Peterson et al. 2011). Para ello se utiliza el algoritmo basado en la teoría de máxima entropía que combina pruebas estadísticas y de probabilidad, con el propósito de estimar las distribuciones de probabilidad, que están subordinadas a restricciones por la información ambiental (Phillips et al. 2006, Elith et al. 2011). Este mismo algoritmo se usa en diferentes campos, como la exploración del suelo, subsuelo y la distribución de especies vegetales (Liu et al. 2017). En relación a lo anterior, uno de los grandes problemas es la dificultad para identificar la extensión de diferentes tipos de vegetación, lo que es un obstáculo para determinar la superficie que ocupan las especies de las zonas áridas, por lo que conocer la distribución permitirá realizar un adecuado manejo de los recursos naturales y la planificación en el uso del suelo ante condiciones ambientales cambiantes (Rehfeldt et al. 2012). Por lo anterior, el objetivo del estudio fue identificar las propiedades físicas y químicas del suelo que permiten la sobrevivencia y la permanencia de las poblaciones de E. antisyphilitica, en dos áreas con vegetación natural del estado de Coahuila.

\section{MATERIALES Y MÉTODOS}

\section{Área de estudio}

Comprende dos áreas del Estado de Coahuila (Figura 1), la primera designada localidad Cuatro Ciénegas, situada en la porción central del estado, con coordenadas $26^{\circ} 58^{\prime} 27.13^{\prime \prime}$ y $26^{\circ} 44^{\prime} 53.56^{\prime \prime} \mathrm{LN}$ y, $102^{\circ} 28^{\prime} 40.70 "$ y $102^{\circ} 15^{\prime} 45.07 "$ LO. La altitud mínima es de 790 m situada en la depresión del Valle de Cuatro Ciénegas, con altitud máxima de $2100 \mathrm{~m}$ en la Sierra la Fragua (INEGI 2006). En la región, 


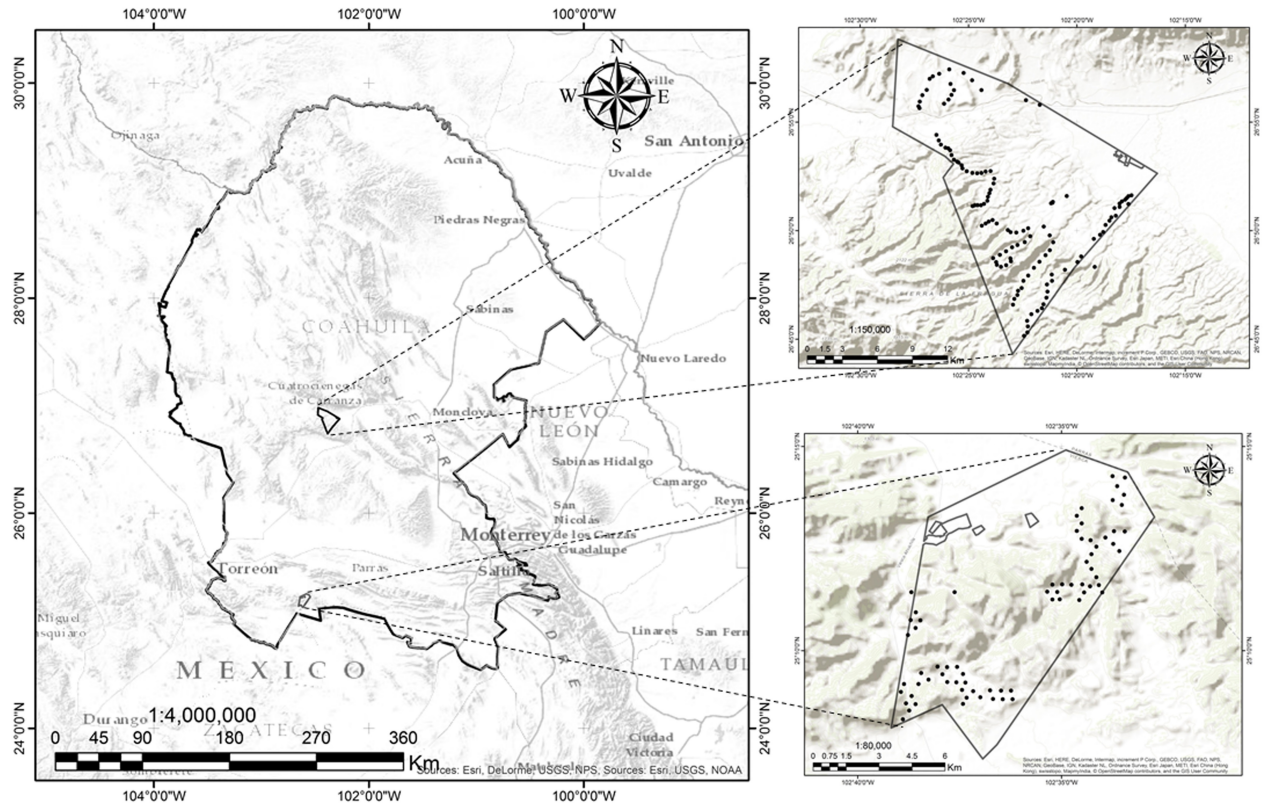

Figura 1. Áreas de estudio y los sitios de muestreo en las localidades Cuatro Ciénegas y Viesca, Coahuila, México.

predomina el tipo de suelo Leptosol calcárico, característico de las áreas cerriles, de lomerío, con textura predominante franco arcillosa. El material geológico es roca sedimentaria caliza, con horizontes someros de profundidad máxima de $20 \mathrm{~cm}$, con limitante del material parental (INEGI 2015). Se presentan dos tipos de clima; muy seco semicálido BWhw, y seco semicálido BSohw. La temperatura mínima es de 6 ${ }^{\circ} \mathrm{C}$, la máxima de $47{ }^{\circ} \mathrm{C}$ con media anual de 21.4 ${ }^{\circ} \mathrm{C}$, y precipitación anual de $245.1 \mathrm{~mm}$, con presencia de lluvias en verano (CONAGUA 2017). La segunda área denominada Localidad Viesca, situada en la porción suroeste del estado de Coahuila, a los $25^{\circ}$ $07^{\prime} 21.10^{\prime \prime}$ y $25^{\circ} 14^{\prime} 52.15^{\prime \prime} \mathrm{LN}$ y, $102^{\circ} 32^{\prime} 50.75^{\prime \prime}$ y $102^{\circ} 39^{\prime} 8.13 "$ LO; tiene una altitud mínima de 1140 $\mathrm{m}$ en los Llanos de Lokhar y máxima de $1880 \mathrm{~m}$ en la Sierra Carolinas (INEGI 1996). En esta zona, el principal tipo de suelo es Leptosol calcáreo en las áreas cerriles, además de Regosol y Calcisol en la bajada de la Sierra Carolinas. La textura predominante es arcillosa, mientras que el material geológico es roca caliza, son suelos someros y profundidad máxima de $50 \mathrm{~cm}$ (INEGI 2015). Tiene clima muy seco semicálido BWhw, con temperatura mínima, máxima y media anual de $-4.4,38.3$ y $18.7{ }^{\circ} \mathrm{C}$, respectivamente; con precipitación anual de $226.3 \mathrm{~mm}$ y régimen de lluvias en verano (CONAGUA 2017).

El tipo de vegetación presente en ambas localidades es matorral desértico rosetófilo, con dominancia de Agave lechuguilla Torr, E. antisyphilitica, Fouquieria splendens Engelm y Jatropha dioica Sessé ex Carv (Encina-Domínguez et al. 2013, INEGI 2014a) y de acuerdo con Villarreal-Quintanilla (2001), la presencia de candelilla se encuentra ampliamente distribuida en las áreas cerriles de los municipios de Cuatro Ciénegas y Viesca, siendo una de las especies dominantes del matorral xerófilo.

\section{Muestreo de vegetación}

Se realizó un muestreo sistemático en gradiente en áreas con presencia de candelilla, realizando evaluaciones en 102 sitios en la localidad Cuatro Ciénegas y 71 en Viesca, la forma del sitio fue un cuadrado de $20 \times 20 \mathrm{~m}$, para tener una superficie de $400 \mathrm{~m}^{2}$ (CONAFOR 2011). En cada sitio se registraron las coordenadas geográficas y se evaluaron in situ las variables: altura, diámetros mayor y menor de los ejemplares de candelilla pre- 
sentes. Adicionalmente, se obtuvieron las características del hábitat, relieve y suelo, así como tipo de matorral, disturbio, presencia de sobrexplotación, presencia de ganado, erosión hídrica o eólica clases diamétricas de la especie, textura al tacto y pedregosidad (PMC 2011). Con las evaluaciones in situ y la revisión de literatura de las condiciones del hábitat y los requerimientos de suelo de la especie, se identificaron las condiciones edáficas que permiten la presencia de la especie, y de acuerdo con De la Garza y Berlanga (1993), Zamora-Martínez et al. (2013), Martínez et al. (2014), y Martínez et al. (2015) se seleccionaron las propiedades del suelo que tienen mayor incidencia en el desarrollo de la candelilla.

\section{Modelación y validación}

Se obtuvieron los archivos tipo ráster (.tif) de la base de datos Soilgrid con resolución espacial de 250 $\mathrm{m}$, del Soil Geographic Databases Compendium (Batjes et al. 2017). Estos archivos fueron desarrollado en el 2016 por el Global Soil Information Facilities (GSIF) del International Soil Reference and Information Centre (ISRIC), los cuales son predicciones espaciales para integrar un sistema automatizado de cartografía del suelo (Hengl et al. 2014, Hengl et al. 2017). En el estudio se usaron nueve propiedades, seis de condiciones físicas y tres de condiciones químicas (Tabla 1).

Los archivos ráster se transformaron a formato ascii (.asc) con el programa Diva Gis 7.5 (Guarino et al. 2002). Posteriormente se procesaron con el método distribución de máxima entropía, empleando el programa Maxent 3.4.1 (Phillips 2008), considerado como el programa más apropiado para la modelación de la distribución de la flora y la fauna (Elith et al. 2006, Phillips 2008). Para la predicción se utilizó el $75 \%$ de los sitios de muestreo $n=91$ en Cuatro Ciénegas y $n=54$ en Viesca. Mientras que el $25 \%$ restante se utilizó para la validación del modelo; con $n=30$ en Cuatro Ciénegas y $n=17$ en Viesca. En cada pronóstico y validación se realizaron los análisis estadístico de las propiedades evaluadas mediante la prueba del área bajo la curva (AUC), la prueba de regularización de Jackknife y la salida geográfica o mapas de la probable distribución de la especie en archivos ráster (Phillips et al. 2017).

Para evaluar el rendimiento de los modelos de presencia-ausencia de especies en un área ecológica se empleó la prueba estadística de Kappa (Allouche et al. 2006), la cual está basada en los errores de omisión y comisión que deriva en un índice de Kappa entre el mapa clasificado y los datos de distribución de la candelilla obtenido en campo, con la finalidad de evaluar la capacidad de la predicción del modelo en la determinación de la presencia de la especie de interés (Jianyu y Pan 2015). Finalmente, los archivos en formato ráster se procesaron en Diva Gis 7.5 para obtener la cartografía de la probabilidad de distribución de la especie.

\section{RESULTADOS}

\section{Evaluación del modelo de predicción}

En los dos predios el AUC de predicción fue cercano a uno, siendo ligeramente superior en la localidad Viesca con valor de 0.973 , en comparación con 0.927 de Cuatro Ciénegas. El comportamiento de las curvas de predicción y de validación se muestran en la Figura 2, el color rojo es la predicción y exhibe el ajuste del modelo a los datos del muestreo, mientras que la curva color azul corresponde a la validación, e indica el grado de ajuste del modelo a los datos de prueba. El valor de concordancia del índice de Kappa fue superior en Viesca con 0.953, mientras que en Cuatro Ciénegas fue de 0.885 .

\section{Contribución y curvas de respuesta}

En la determinación de la importancia en la probabilidad de distribución, con el porcentaje y el nivel de influencia que contribuyen al modelo; para la localidad Cuatro Ciénegas, las propiedades más importantes fueron; profundidad del suelo, densidad aparente (DA), capacidad de intercambio catiónico (CIC) y contenido de carbono orgánico (COS), sumando el $89.3 \%$ de la influencia en el modelo, mientras que el faltante se distribuye en las cinco propiedades restantes. En la localidad Viesca, las propiedades más influyentes fueron profundidad, DA y pedregosidad sumando estos aspectos físicos el 
Tabla 1. Las variables físicas y químicas del suelo utilizadas en la modelación y su contribución al modelo.

\begin{tabular}{llcccc}
\hline \multicolumn{1}{c}{ Variables } & \multicolumn{1}{c}{ Unidad } & $\begin{array}{c}\text { Valores } \\
\text { promedio }^{1}\end{array}$ & $\begin{array}{c}\text { Valores } \\
\text { promedio }^{2}\end{array}$ & $\begin{array}{c}\text { Contribución al } \\
\text { modelo }^{1}(\%)\end{array}$ & $\begin{array}{c}\text { Contribución al } \\
\text { modelo }^{2}(\%)\end{array}$ \\
\hline Profundidad absoluta hasta el lecho de roca & $\mathrm{cm}$ & 32.2 & 12.4 & 41.7 & 31.4 \\
Densidad aparente & $\mathrm{kg} \mathrm{m}^{-3}$ & 1449.5 & 1473.8 & 20.8 & 28.1 \\
Contenido de arcillas (0-2 micrómetros) & Fracción de masa en porcentaje & 39.9 & 42.11 & 6 & 2.1 \\
Contenido de limos (2-50 micrómetros) & Fracción de masa en porcentaje & 27.7 & 26.2 & 1.7 & 5.6 \\
Contenido de arenas (50-200 micrómetros) & Fracción de masa en porcentaje & 32.4 & 31.6 & 2.4 & 0.1 \\
Fragmentos gruesos volumétricos (piedras) & Porcentaje & 39.9 & 41.7 & 0.4 & 22.3 \\
Capacidad de intercambio catiónico & $\mathrm{cmol} \mathrm{kg}^{-1}$ & 22.1 & 23.8 & 14.7 & 0.6 \\
Contenido de carbono orgánico en el suelo & $\mathrm{g} \mathrm{kg}^{-1}$ & 10.2 & 6.98 & 12.1 & 8.0 \\
pH del suelo & & 6.86 & 7.02 & 0.2 & 1.6 \\
\hline
\end{tabular}

1 = Localidad Cuatro Ciénegas; 2 = Localidad Viesca.
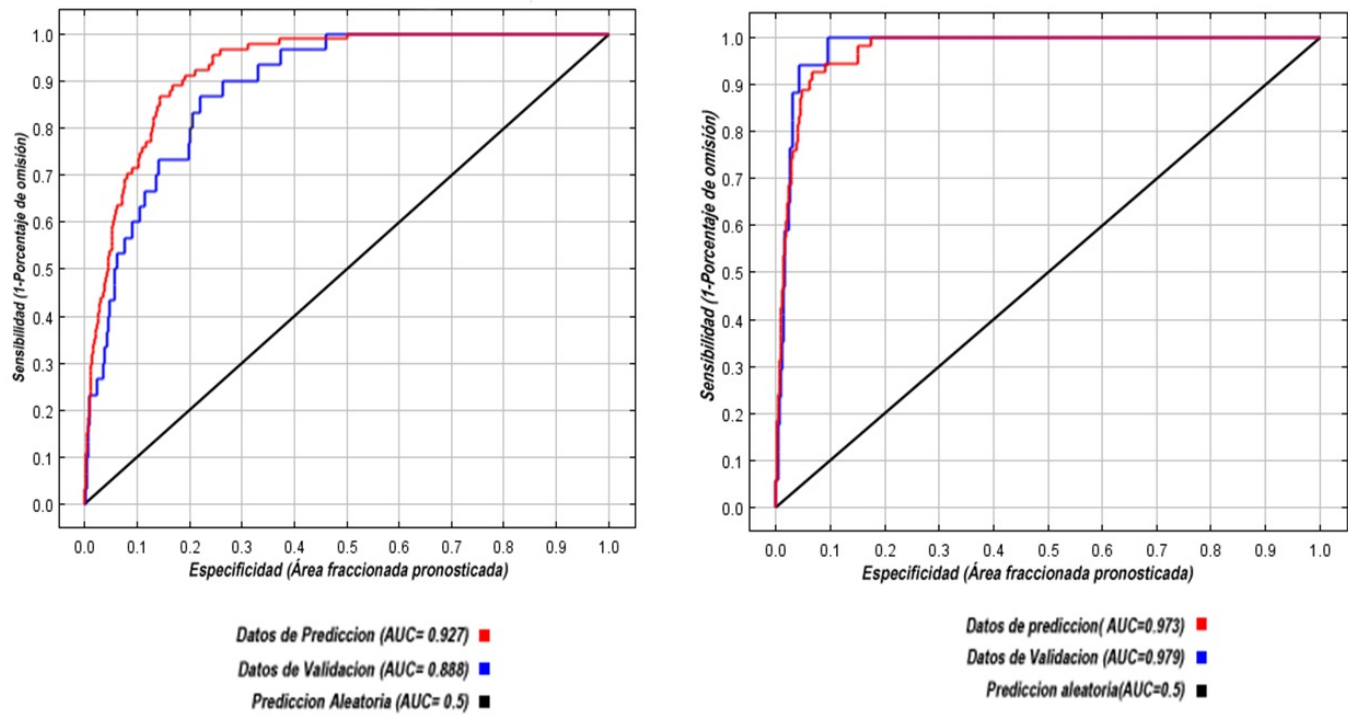

Figura 2. Curva operacional para dos grupos de datos: de predicción en color rojo y validación en color azul, así como el AUC en los predios. a: localidad Cuatro Ciénegas y b: localidad Viesca.

$81.8 \%$ de su contribución al modelo (Tabla 1). En la curva de respuesta hasta con $40 \%$ de contenido de rocas existe alta probabilidad de la presencia de $E$. antisyphilitica, pero con contenido de rocas superior al $51 \%$, disminuye la probabilidad (Figura $3 a$ ).

\section{Salida Geográfica}

El algoritmo de Maxent generó la distribución de probabilidad en el rango de 0.0 a 1.0 , sobre los pixeles de cada archivo ráster: 0.0 indica ausencia y 1.0 presencia de la especie. Se establecieron niveles de aptitud de acuerdo a la probabilidad, donde se consideró aptitud alta (0.71-0.97), media (0.51-0-70), baja (0.31-0.50) y condiciones con nula presencia de candelilla $(0.00-0.30)$, lo que permite identificar las áreas de distribución actual de la especie en estudio. Con la salida del mapa representa el modelo de predicción de las áreas geográficas de la distribución de la especie evaluada, donde son las condiciones ecológicamente realistas para la especie, en este caso condicionadas por factores del suelo como se observa en la Figura 4.

\section{DISCUSIÓN}

\section{Evaluación del modelo de predicción}

Los valores AUC de predicción resultaron apropiados para explicar la distribución de la can- 

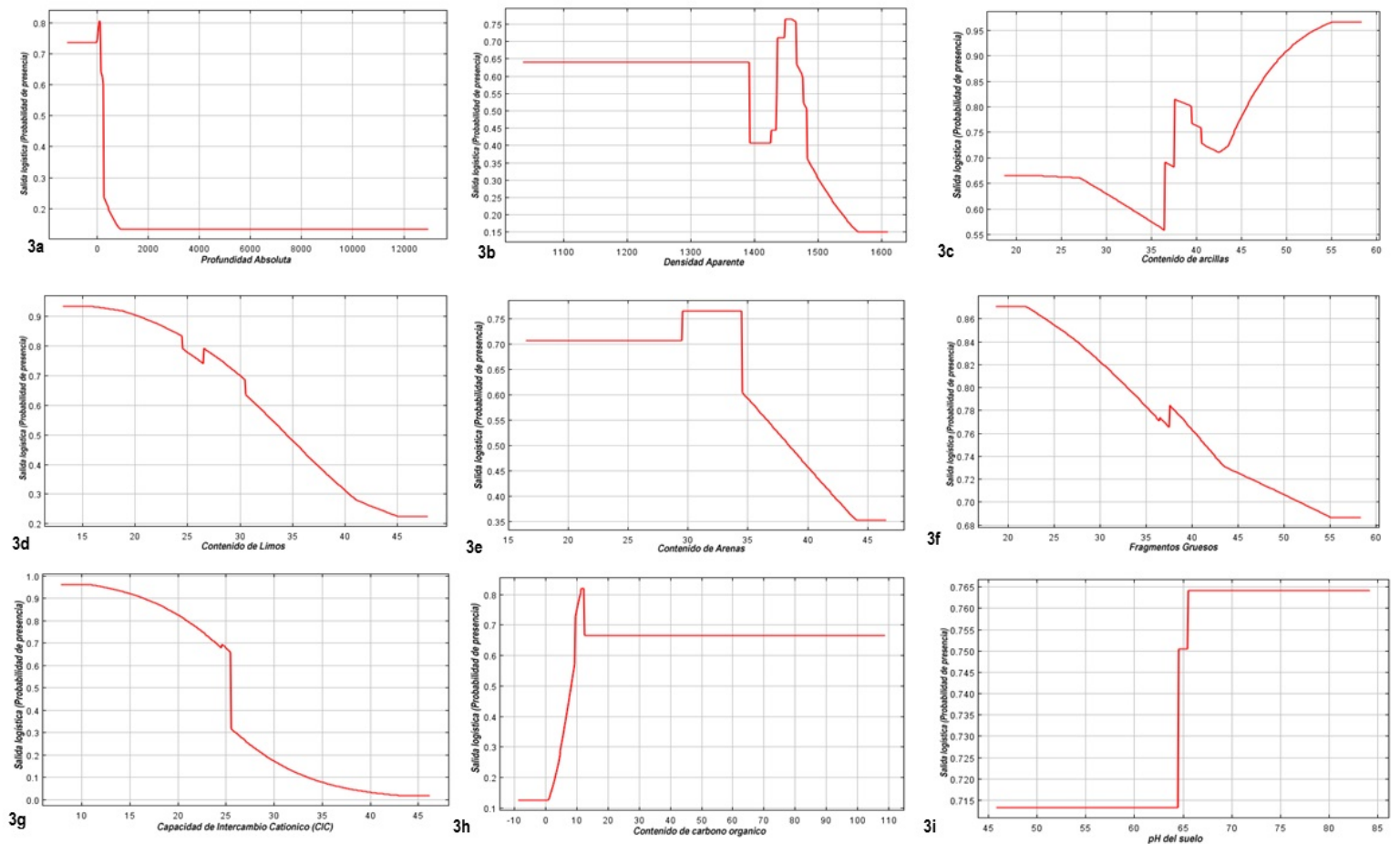

Figura 3. Curvas de respuesta generada para las variables del suelo en localidad Cuatro Ciénegas. profundidad 3a, densidad aparente $3 \mathrm{~b}$, contenido de arcillas $3 \mathrm{c}$, contenido de limo $3 \mathrm{~d}$, contenido de arenas $3 \mathrm{e}$, fragmentos gruesos $3 \mathrm{f}, \mathrm{CIC} 3 \mathrm{~g}, \cos 3 \mathrm{~h}$ y $\mathrm{pH} 3 \mathrm{i}$.
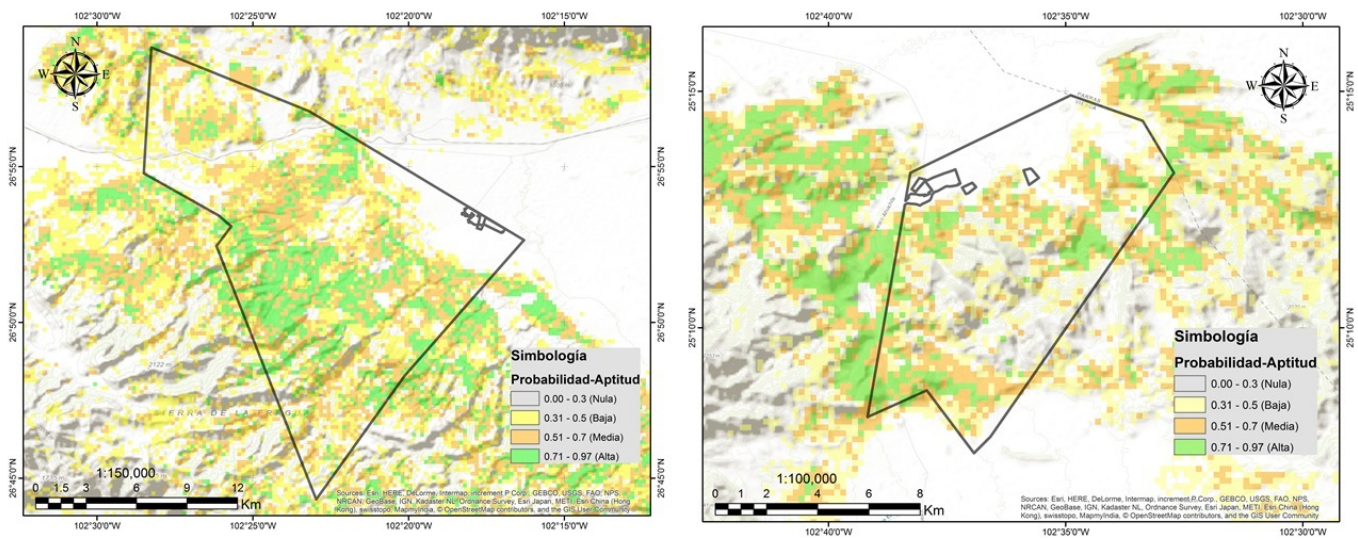

Figura 4. Los mapas de distribución de la candelilla generados por Maxent, en las áreas de planicie no existen poblaciones, mientras que la presencia de la especie está condicionada a la zona cerril en las dos localidades estudiadas.

delilla, lo que se considera como un rendimiento excelente, este indicador mide la capacidad de las predicciones del modelo, por lo tanto, es de utilidad para determinar la presencia o ausencia de la especie, entre más cercano sea a 1 el valor del indicador, se considera con mayor confiabilidad (West et al. 2016). Aunque los valores del índice de Kappa fueron ligeramente menores al AUC, se consideran con una precisión adecuada para determinar la clasificación y la presencia de la candelilla (Li y Guo 2013). Como complemento se han desarrollado los modelos y programas para la valoración de las funciones de los suelos, así como técnicas para la evaluación de los suelos y categorización para suelos naturales y 
antropogénicos (TUSEC) y Soil \& Environment, que también utilizan información de clima, condiciones del relieve, además de información del uso de la tierra que permitirían fortalecer el modelo de predicción de la candelilla (Gallegos-Tavera et al. 2014, BedollaOchoa et al. 2018).

\section{Contribución y curvas de respuesta}

Los suelos presentan alta funcionalidad ecológica, que deben ser considerada en la planeación y manejo de la vegetación natural. Adicionalmente, Bautista et al. (2016) destacan que en la búsqueda de soluciones a la crisis mundial ambiental, es necesario e impostergable, fortalecer el conocimiento de los suelos. Para las propiedades de suelo evaluadas, se comprobó que la profundidad determina la distribución de la candelilla en ambas localidades, teniendo como limitante el escaso suelo y la presencia del afloramiento rocoso, ya que los suelos del grupo Leptosoles son muy superficiales, delgados con enraizamiento restringido a una profundidad menor de $25 \mathrm{~cm}$ (Lehmann 2006). Al respecto Rojas-Molina et al. (2011) y Martínez et al. (2014) indican que la candelilla se desarrolla en laderas de zonas cerriles, con suelos pocos profundos, ricos en carbonatos de calcio, por lo que la curva de respuesta (Figura 3a), registró mayor probabilidad en capas del suelo menores de $50 \mathrm{~cm}$, debido a que el material geológico facilita la percolación conformando el agua subterránea (Salameh et al. 2018). Además, el sustrato de origen calcáreo puede albergar una amplia diversidad de plantas, por lo que el material parental es un reservorio de humedad y de nutrientes, donde se dan los procesos homeostáticos de regulación del ecosistema, siendo estas condiciones el hábitat idóneo para el desarrollo de la candelilla (Augusto et al. 2017, Pittarello et al. 2017).

La DA fue el segundo parámetro físico de importancia en las dos localidades, se considera que tiene relación con propiedades y procesos del suelo, como las propiedades hidráulicas, la compactación del suelo, el contenido de materia orgánica y la erosión (Beutler et al. 2017). Hay mayor probabilidad de que la especie se localice en el rango de 1 420 a $1480 \mathrm{~kg} \mathrm{~m}^{-3}$ (Figura 3b) al acercarse a valor critico $1690 \mathrm{~kg} \mathrm{~m}^{-3}$ representa una restricción para el establecimiento y sobrevivencia de la especie en condiciones naturales, ya que Da afecta la infiltración del agua, las restricciones de enraizamiento, la capacidad de agua disponible, la porosidad del suelo, la disponibilidad de elementos nutritivos y favorece la actividad de los microorganismos del suelo que tienen impacto en procesos como la meteorización y productividad de las plantas (Dexter 2004, Padilla et al. 2017)

La pedregosidad fue la tercer propiedad de relevancia en Viesca, al respecto Zamora-Martínez et al. (2013) mencionan que esta especie se establece en suelos con presencia de piedras, con buen drenaje y ricos en carbonato de calcio, con valores en el rango de 31 a 60\% (INEGI 2014b). La presencia de fragmentos gruesos mayores de $6 \mathrm{~cm}$ de diámetro denominadas piedras, son condiciones desfavorables para otras especies vegetales, debido a que la pedregosidad favorece mayor acumulación de agua en el suelo y la roca, por lo que ofrece humedad disponible para el desarrollo de las plantas que sobreviven en los ambientes áridos (Salas-Pascual et al. 2017). En contraparte una desventaja, de suelos superficiales y con cantidades considerables de piedras tienen alta fragilidad a la erosión, lo que incrementa el riesgo de afectación al ecosistema, siendo la extracción de las plantas completas el factor que incrementa el riesgo de pérdida de suelo (MartínezBallesté y Mandujano 2013).

La CIC fue la tercera característica en importancia solo en Cuatro Ciénegas, con valor de 22.1 $\mathrm{cmol} \mathrm{kg}^{-1}$, contribuyendo de manera importante al modelo de predicción en un $14.7 \%$, en tanto en Viesca, con un valor ligeramente mayor de $23.8 \mathrm{cmol}$ $\mathrm{kg}^{-1}$, solo aportó el $0.6 \%$ al modelo. La diferencia en los valores de la CIC, se debe al tipo de textura que predomina en cada región, ya que la CIC es afectada por la cantidad de arcillas y los contenidos de materia orgánica presentes, lo que indica que a mayor contenido de arcillas y de materia orgánica los suelos tienen mayor potencial para retener e intercambiar elementos nutritivos en forma iónica (Asadzadeh et al. 2019). La curva de respuesta de la CIC (Figura $3 \mathrm{~g}$ ) indica que hay mayor probabilidad de presencia 
de la candelilla en el rango de 10 a $24 \mathrm{cmol} \mathrm{kg}^{-1}$, disminuyendo drásticamente la probabilidad de que la especie estudiada pueda encontrarse a valores mayores de $25 \mathrm{cmol} \mathrm{kg}^{-1}$, lo que indica que la candelilla tiene su hábitat en suelos con altos contenidos de arcilla pero bajos niveles de materia orgánica, por lo que derivado de un incremento en la CIC habrá una mayor disponibilidad de humedad en el suelo y de retención de nutrientes, debido a la disminución de la DA y al incremento en el contenido de materia orgánica del suelo (Olmo et al. 2016). Al respecto Park et al. (2019) señalan que las arcillas poseen la capacidad de retener o intercambiar cationes, $\mathrm{Ca}^{+2}$, $\mathrm{Mg}^{+2}, \mathrm{~K}^{+}, \mathrm{Na}^{+}$, en función de factores como tamaño de la partícula, contenido de materia orgánica y cantidad de minerales arcillosos, además de que su CIC oscila de 10 a $150 \mathrm{cmol} \mathrm{kg}^{-1}$, rango que superó a los valores determinados en ambas regiones y que oscilaron de 22.1 y $23.8 \mathrm{cmol} \mathrm{kg}^{-1}$, los cuales potencialmente se deben a la erosión, que favorece la pérdida de las arcillas, así como la reducción de la disponibilidad de los elementos esenciales para el desarrollo de las plantas, y en consecuencia en la capacidad productiva de los ecosistemas (Krasilnikov et al. 2013).

El COS fue considerado como la cuarta propiedad que determinó la distribución de la candelilla en ambas localidades. Al respecto Alcántara et al. (2016), mencionan que el subsuelo de las áreas forestales tienen un alto contenido de carbono orgánico. Los valores en ambas localidades fueron coincidentes con las cantidades obtenidas en las áreas de pastizal y vegetación natural con valores máximos de 13.2 a $9.60 \mathrm{~g} \mathrm{~kg}^{-1}$, respectivamente (Saha et al. 2011). Por lo tanto, en función de la curva de respuesta para COS es más probable encontrar la candelilla entre 1.0 y 14 de COS, condición que concuerda con las cantidades reportadas en especies desérticas y de matorral con valores de 3.4 a 16.8 $\mathrm{g} \mathrm{kg}^{-1}$ (Wang et al. 2015). Generalmente los contenidos de COS se encuentran en los primeros $20 \mathrm{~cm}$ de profundidad del suelo (Lai et al. 2013), característica que presenta el tipo de suelo donde se desarrolla, de manera adecuada, la candelilla. En contra parte Aryal et al. (2018), destacan que en México es indispensable la conservación y el aprovechamiento sostenible de las áreas de distribución de la candelilla, evitando el pastoreo, los incendios y sobreexplotación ya que estas actividades afectan los contenidos de COS, provocando incrementos en la mineralización, lixiviación y erosión, procesos que repercuten en las propiedades físicas y químicas de los suelos. Para los componentes de la textura del suelo, la mayor probabilidad de presencia de la candelilla se encuentra de 17 a $35 \%$ de contenido de arena, para el contenido de limo la probabilidad más alta osciló de 13 a 25\% (Figura 3e), la probabilidad de presencia de esta especie es mayor con contenidos de arcilla del 35 al $57 \%$, preferentemente en textura franca a arcillosa, ya que los minerales de arcilla contribuyen a la formación de los agregados (Karup et al. 2016) y la textura favorece la permeabilidad, la retención del agua, de los nutrientes originado por la biodisponibilidad de materia orgánica del suelo, donde influye el tamaño de las partículas o agregados (Bimüller et al. 2016).

\section{Salida Geográfica}

Para la predicción de distribución de la candelilla fue importante elegir las propiedades más sobresalientes, que fortalecen los modelos de predicción, para realizar la extrapolación de la modelación a nivel municipal y estatal, lo que representa una herramienta adecuada para determinar la situación actual y futura de los recursos naturales de los ecosistemas desérticos (Hageer et al. 2017). En la modelación del suelo es importante evaluar este componente desde seis funciones principales, una como sustento de la vegetación, entendiendo que los factores físicos y químicos del suelo favorecen la presencia o ausencia de una especie vegetal (Lehmann y Stahr 2010). Adicionalmente, como lo mencionan Lehmann et al. (2008), para la evaluación de la presencia de una especie en determinadas condiciones de suelo es necesario la entrada de datos primarios del suelo e información de la presencia de la especie y finalmente el proceso estadístico o de evaluación que permitiré obtener información confiable, en este caso el uso de Maxent fue una alternativa confiable.

El uso de la modelación de la distribución de la 
candelilla, permite realizar trabajos en la vegetación de zonas áridas, además de otros ecosistemas donde se pueda analizar el rango de distribución de especies de importancia económica o ecológica, los análisis ecológicos entre las variables climáticas, del suelo y la distribución de las plantas con la finalidad de generar información de áreas o hábitats idóneos para las especies vegetales (Sahragard et al. 2018). Además, el empleo del Maxent, con énfasis en especies que tienen o tendrán afectaciones por el cambio climático y la posible pérdida de su hábitat y de los ejemplares de plantas, representa una alternativa para evaluar cambios en el hábitat de los vegetales, mediante estudios de vulnerabilidad por medio de la modelación (Foden et al. 2019). Derivado del presente trabajo, se recomienda evaluar la metodología de Máxima Entropía para conocer la distribución actual y a futuro de las poblaciones naturales de la candelilla en condiciones ambientales utilizando variables como temperaturas y precipitación, además de someter a la reflexión el impacto de la actividad de extracción de ejemplares vivos de can- delilla que puede tener efectos negativos en la permanencia de la especie, para saber si se incrementa el riesgo de afectación directa al ecosistema por el método de cosecha utilizado, el cual consiste en la extracción de plantas completas.

\section{CONCLUSIONES}

En Cuatro Ciénegas, las propiedades del suelo que permiten la distribución y supervivencia de la candelilla, fueron la profundidad, densidad aparente, la capacidad de intercambio catiónico y el contenido de carbono orgánico, mientras en Viesca, influyeron la profundidad, densidad aparente y pedregosidad. Por lo que los factores físicos del suelo determinan en gran medida la presencia y la distribución de las poblaciones de la candelilla en ambas localidades son: la profundidad, densidad aparente y el porcentaje de fragmentos gruesos volumétricos y en cuanto a los factores químicos destacan la capacidad de intercambio catiónico y el contenido de carbono orgánico en el suelo.

\section{LITERATURA CITADA}

Alcántara V, Don A, Well R, Nieder R (2016) Deep ploughing increases agricultural soil organic matter stocks. Global change biology 22: 2939-2956.

Allouche O, Tsoar A, Kadmon R (2006) Assessing the accuracy of species distribution models: Prevalence, kappa and the true skill statistic (TSS). Journal of Applied Ecology 43: 1223-1232.

Arato M, Speelman S, Van Huylenbroeck G (2014) The contribution of non-timber forest products towards sustainable rural development: The case of candelilla wax from the Chihuahuan Desert in Mexico. Natural Resources Forum 38: 141-153.

Aryal D, Morales-Ruiz D, Tondopó-Marroquín C, Pinto-Ruiz R, Guevara-Hernández F, Venegas-Venegas J, Ley de Coss A (2018) Soil organic carbon depletion from forests to grasslands conversion in Mexico: A Review. Agriculture 8(11): 181 Doi: 10.3390/agriculture8110181.

Asadzadeh F, Maleki-Kakelar M, Shabani F (2019) Predicting cationic exchange capacity in calcareous soils of East-Azerbaijan province, northwest Iran. Communications in Soil Science and Plant Analysis 50: 11061116.

Augusto L, Achat DL, Jonard M, Vidal D, Ringeval B (2017) Soil parent material-a major driver of plant nutrient limitations in terrestrial ecosystems. Global Change Biology 23: 3808-3824.

Bautista F, Gallegos A, Pacheco A (2016) Análisis de las funciones ambientales de los suelos con datos de perfiles (Soil \& Environment). Skiu. México. 72p.

Batjes NH, Ribeiro E, Oostrum AV, Leenaars J, Hengl T, Mendes de Jesus J (2017) WoSIS: Providing standardised soil profile data for the world. Earth System Science Data 9: 1-14. 
Bedolla-Ochoa C, Bautista F, Gallegos A (2018) Environmental functions of smallholder farmer land classes in the Zicuirán-Infiernillo Biosphere Reserve, Mexico. Revista Chapingo Serie Ciencias Forestales y del Ambiente 24: 265-274.

Beutler SJ, Pereira MG, Tassinari WDS, Menezes MDD, Valladares GS, Anjos LHCD (2017) Bulk density prediction for histosols and soil horizons with high organic matter content. Revista Brasileira de Ciencia do Solo 41: e0160158. Doi: 10.1590/18069657rbcs20160158.

Bimüller C, Kreyling O, Kölbl A, von Lützow M, Kögel-Knabner I (2016) Carbon and nitrogen mineralization in hierarchically structured aggregates of different size. Soil and Tillage Research 160: 23-33.

CONAFOR (2011) Manual y procedimientos para el muestreo de campo. Remuestreo 2011. Inventario Nacional Forestal y de Suelos. Comisión Nacional Forestal. SEMARNAT. Zapopan, Jalisco, México. 140p.

CONAGUA (2017) Normales Climatológicas por Estación. Ciudad de México, México. http://smn.cna.gob.mx/es. Fecha de consulta: 1 de octubre de 2017.

De la Garza F, Berlanga C (1993) Metodología para la evaluación y manejo de candelilla en condiciones naturales. Folleto Técnico Número 5. Campo Experimental La Sauceda INIFAP. 46p.

Dexter AR (2004) Soil physical quality Part I. Theory, effects of soil texture, density, and organic mailer, and effects on root growth. Geoderma 120: 201-214.

Elith J, Graham CH, Anderson RP, Dudík M, Ferrier S, Guisan A, et al. (2006) Novel methods improve prediction of species' distributions from occurrence data. Ecography 29: 129-151.

Elith J, Phillips SJ, Hastie T, Dudík M, Chee YE, Yates CJ (2011) A statistical explanation of MaxEnt for ecologists. Diversity and Distributions 17: 43-57.

Encina-Domínguez JA, Meave JA, Zarate-Lupercio A (2013) Structure and woody species diversity of the Dasylirion cedrosanum (Nolinaceae) rosette scrub of central and outhern Coahuila State, México. Botanical Sciences 91: 335-347.

Foden WB, Young BE, Akçakaya HR, Garcia RA, Hoffmann AA, Stein BA Hole DG (2019) Climate change vulnerability assessment of species. Wiley Interdisciplinary Reviews: Climate Change 10(1) e551. Doi: 10.1002/wcc.551.

Gallegos-Tavera Á, Bautista F, Álvarez O (2014) Software para la evaluación de las funciones ambientales de los suelos (Assofu). Revista Chapingo Serie Ciencias Forestales y del Ambiente 20: 237-249.

Guarino L, Jarvis A, Hijmans RJ, Maxted N (2002) Geographic Information Systems (GIS) and the conservation and use of plant genetic resources. In: Engels JMM, Ramanatha-Rao V, Brown AHD, Jackson MT (eds.) Managing plant genetic diversity. International Plant Genetic Resources Institute. Roma, Italia. pp: 387-404.

Hageer Y, Esperón-Rodríguez M, Baumgartner JB, Beaumont LJ (2017) Climate, soil or both? Which variables are better predictors of the distributions of Australian shrub species?. PeerJ 5: e3446. Doi: 10.7717/peerj. 3446.

Hengl T, Mendes de Jesus J, MacMillan RA, Batjes NH, Heuvelink GBM, Ribeiro E, et al. (2014) SoilGrids1km - Global soil information based on automated mapping. PLOS ONE 9: e105992. Doi: 10.1371/journal.pone.0105992.

Hengl T, Mendes de Jesus J, Heuvelink GBM, Ruiperez-Gonzalez M, Kilibarda M, Blagotić A, et al. (2017) SoilGrids250m: Global gridded soil information based on machine learning. PLOS ONE 12: e0169748. Doi: $10.1371 /$ journal.pone.0169748. 
INEGI (1996) Conjunto de datos vectoriales de la carta Topográfica Bajío de Ahuichila G13D48. Escala 1:50 000. Aguascalientes, Ags, Mexico. http://www.beta.inegi.org.mx/app/mapas. Fecha de consulta: 27 de septiembre de 2017.

INEGI (2006) Conjunto de datos vectoriales de la carta Topográfica Rosario Viejo G13B58. Escala 1:50 000. Aguascalientes, Ags, Mexico. http://www.beta.inegi.org.mx/app/mapas. Fecha de consulta: 27 de septiembre de 2017.

INEGI (2014a) Datos vectoriales uso de suelo y vegetación serie $V$ escala 1:250 000. Aguascalientes, Ags, México. http://www.inegi.org.mx/geo/contenidos/recnat/usosuelo. Fecha de consulta: 28 de septiembre de 2017.

INEGI (2014b) Diccionario de Datos Edafológicos escala 1: 250, 000. Aguascalientes, Ags, México. http://www. inegi.org.mx/geo/contenidos/recnat/edafologia/doc/dd_edafologicos_v3_250k.pdf. Fe-cha de consulta 29 de septiembre de 2017.

INEGI (2015) Conjunto de datos vectorial edafológico, escala 1: 250 000, Serie II Continuo Nacional. Aguascalientes, Ags, México. http://www.inegi.org.mx/geo/contenidos/recnat/edafologia/vectorial_serieii.aspx. Fecha de consulta 29 de septiembre de 2017.

Jianyu RGC, Pan Y (2015) The impact of positional errors on soft classification accuracy assessment: A simulation analysis. Remote Sensing 7: 579-599.

Karup D, Moldrup P, Paradelo M, Katuwal S, Norgaard T, Greve MH, et al. (2016) Water and solute transport in agricultural soils predicted by volumetric clay and silt contents. Journal of Contaminant Hydrology 192: 94-202.

Krasilnikov P, Gutiérrez-Castorena M del C, Ahrens RJ, Cruz-Gaistardo CO, Sedov S, Solleiro-Rebolledo E (2013) The Soil of Mexico: Netherlands, Springer 187p.

Lai L, Li Y, Tian Y, Jiang L, Zhao X, Zhu L, et al. (2013) Effects of added organic matter and water on soil carbon sequestration in an arid region. PLOS ONE 8: e70224. Doi: 10.1371/journal.pone.0070224.

Lehmann A (2006) Technosols and other proposals on urban soils for the WRB (World Reference Base for Soil Resources). International Agrophysics 20: 129-134.

Lehmann A, David S, Stahr K (2008) TUSEC.-Technique for soil evaluation and categorization for natural and anthropogenic soils. Universitat Hohenheim Bodenkundliche Hefte 86. Alemania. 224p.

Lehmann A, Stahr K (2010) The potential or soil functions and planner-oriented soil evaluation to archive sustainable land use. Soils and Sediments 10: $1092-1102$

Li W, Guo Q (2013) How to assess the prediction accuracy of species presence-absence models without absence data? Ecography 36: 788-799.

Liu Y, Zhou K, Xia Q (2017) A MaxEnt model for mineral prospectivity mapping. Natural Resources Research 27: 299-313.

Martínez-Ballesté A, Mandujano MC (2013) The consequences of harvesting on regeneration of a non-timber wax producing species (Euphorbia antisyphilitica Zucc.) of the Chihuahuan Desert. Economic Botany 67: 121-136.

Martínez SM, Hermosillo-Rojas DE, Mojica-Guerrero AS (2015) Potencial productivo y zonificación para el uso y manejo de especies forestales de zonas áridas. Primera Edición. INIFAP. Campo Experimental La Campana. Chihuahua, México. 119p. 
Martínez SM, Hermosillo-Rojas DE, Mojica-Guerrero AS (2014) Guía para la zonificación forestal de zonas áridas de México. Publicación Especial 12. INIFAP. Campo Experimental La Campana. Chihuahua, México. 29p.

Muñoz-Ruiz CV, López-Díaz S, Covarrubias-Villa F, Villar-Luna E, Medina-Medrano JR, Barriada LG (2016) Effect of abiotic stress conditions on the wax production in candelilla (Euphorbia antisyphilitica zucc). Revista Latinoamericana de Química 1: 26-33.

Olmo M, Villar R, Salazar P, Alburquerque JA (2016) Changes in soil nutrient availability explains biochar's impact on wheat root development. Plant and Soil 399: 333-343.

Owens HL, Campbell LP, Dornak LL, Saupe EE, Barve N, Soberón J, et al. (2013) Constraints on interpretation of ecological niche models by limited environmental ranges on calibration areas. Ecological Modelling 263: 10-18.

Padilla FM, Peña-Fleitas MT, Fernández MD, Del Moral F, Thompson RB, Gallardo M (2017) Responses of soil properties, crop yield and root growth to improved irrigation and $\mathrm{N}$ fertilization, soil tillage and compost addition in a pepper crop. Scientia Horticulturae 225: 422-430.

Park SM, Alessi DS, Baek K (2019) Selective adsorption and irreversible fixation behavior of cesium onto 2: 1 layered clay mineral: A mini review. Journal of Hazardous Materials 369: 569-576.

Peterson AT, Soberón J, Pearson RG, Anderson RP, Martínez-Meyer E, Nakamura M, et al. (2011) Ecological niches and geographic distributions. Princeton University Press. Princeton, New Jersey, USA. 328p.

Phillips S (2008) A brief tutorial on Maxent. AT\&T Labs-Research. Center for Biodiversity and Conservation at the American Museum of Natural History. New York, USA. http://biodiversityinformatics.amnh.org/open_source/ maxent/. Fecha de consulta: 1 de septiembre de 2017.

Phillips SJ, Anderson RP, Dudík M, Schapire RE, Blair ME (2017) Opening the black box: an open-source release of Maxent. Ecography 40: 887-893.

Phillips SJ, Anderson RP, Schapire RE (2006) Maximum entropy modeling of species geographic distributions. Ecological Modelling 190: 231-259.

Pittarello M, Lonati M, Gorlier A, Probo M, Lombardi G (2017) Species-rich Nardus stricta grasslands host a higher vascular plant diversity on calcareous than on siliceous bedrock. Plant Ecology \& Diversity 10: 343351.

PMC (2011) Manual de Referencia para el Muestreo Semicuantitativo del Inventario Forestal Estatal Cuantitativo: Matorrales. Texcoco, Estado de México, México. http://pmcarbono.org/pmc/descargas/manuales/Manual_ Inventario_Forestal_Estatal_Semicuantitativo_2_Matorrales.pdf. Fecha de consulta: 10 de septiembre de 2017.

Rehfeldt GE, Crookston NL, Sáenz-Romero C, Campbell EM (2012) North American vegetation model for landuse planning in a changing climate: A solution to large classification problems. Ecological Applications 22: 119-141.

Rojas-Molina R, Saucedo-Pompa S, De León-Zapata MA, Jasso-Cantú D, Aguilar CN (2011) Pasado, presente y futuro de la candelilla. Revista Mexicana de Ciencias Forestales 2: 7-18.

Saha D, Kukal SS, Sharma S (2011) Land use impacts on SOC fractions and aggregate stability in typic ustochrepts of Northwest India. Plant and Soil 339: 457-470.

Sahragard HP, Ajorlo M, Karami P (2018) Modeling habitat suitability of range plant species using random forest method in arid mountainous rangelands. Journal of Mountain Science 15: 2159-2171. 
Salas-Pascual M, Fernández-Negrín E, Quintana-Vega G, Del Arco-Aguilar M (2017) Efecto de la inclinación y pedregosidad sobre el reparto del agua de lluvia, su cuantificación y aplicación al estudio de la vegetación en zonas áridas. Investigaciones Geográficas Boletín del Instituto de Geografía 92: 51-63.

Salameh E, Toll M, AI Raggad M (2018) Hydrogeochemical prospecting for evaporates and clay deposits in Harrat ash Shaam basalts, Jordan. Journal of Geochemical Exploration 186: 243-255.

Villa-Castorena M, Catalán-Valencia EA, Inzunza-Ibarra MA, González-López M de L, Arreola- Ávila JG (2010) Producción de plántulas de candelilla (Euphorbia antisyphilitica Zucc.) mediante estacas. Revista Chapingo. Serie Ciencias Forestales y del Ambiente 16: 37-47.

Villarreal-Quintanilla JÁ (2001) Listados florísticos de México XXIII. Flora de Coahuila. Instituto de Biología. México. 138p.

Wang X, Wang J, Xu M, Zhang W, Fan T, Zhang J (2015) Carbon accumulation in arid croplands of northwest China: Pedogenic carbonate exceeding organic carbon. Scientific Reports 5: 11439. Doi: 10.1038/srep114 39.

West AM, Kumar S, Brown CS, Stohlgren TJ, Bromberg J (2016) Field validation of an invasive species Maxent model. Ecological Informatics 36: 126-134.

Zamora-Martínez MC, Méndez-Espinoza C, Pérez-Miranda R, Cortés-Barrera EN (2013) Euphorbia antisyphilitica Zucc.: Recurso forestal no maderable de alto valor económico. Folleto Técnico 12. CENID-COMEP, INIFAP. México. 64p. 
